Probability, Networks and Algorithms von Neumann algebras

R.M. Brouwer 
CWI is the National Research Institute for Mathematics and Computer Science. It is sponsored by the Netherlands Organization for Scientific Research (NWO).

CWI is a founding member of ERCIM, the European Research Consortium for Informatics and Mathematics.

CWI's research has a theme-oriented structure and is grouped into four clusters. Listed below are the names of the clusters and in parentheses their acronyms.

\section{Probability, Networks and Algorithms (PNA)}

Software Engineering (SEN)

Modelling, Analysis and Simulation (MAS)

Information Systems (INS)

Copyright (C) 2001, Stichting Centrum voor Wiskunde en Informatica

P.O. Box 94079, 1090 GB Amsterdam (NL)

Kruislaan 413, 1098 SJ Amsterdam (NL)

Telephone +31205929333

Telefax +31205924199

ISSN 1386-3711 


\title{
A Bicategorical Approach to Morita Equivalence for von Neumann Algebras
}

\author{
R.M. Brouwer \\ $C W I$ \\ P.O. Box 94079, 1090 GB Amsterdam, The Netherlands
}

\begin{abstract}
We relate Morita equivalence for von Neumann algebras to the "Connes fusion" tensor product between correspondences. In the purely algebraic setting, it is well known that rings are Morita equivalent if and only if they are equivalent objects in a bicategory whose 1-cells are bimodules. We present a similar result for von Neumann algebras. We show that von Neumann algebras form a bicategory, having Connes's correspondences as 1-morphisms, and (bounded) intertwiners as 2-morphisms. Further, we prove that two von Neumann algebras are Morita equivalent if and only if they are equivalent objects in the bicategory. The proofs make extensive use of the Tomita-Takesaki modular theory.
\end{abstract}

2000 Mathematics Subject Classification: 46L99, 18D05

Keywords and Phrases: Morita equivalence, von Neumann algebras, bicategories

Note: This report will appear in the Journal of Mathematical Physics, 2003. The main part of this work was

done as a master's thesis at the Korteweg-de Vries Instituut, University of Amsterdam.

\section{INTRODUCTION}

A recent trend in the interaction between operator algebras and quantum physics is the appearance of the notion of Morita equivalence [17] in both string theory [20] and quantization theory [18, 13]. Furthermore, the tensor product between so-called correspondences of von Neumann algebras constructed by Connes [7] and Sauvageot [19] has played a useful role in conformal field theory [24] as well as in the theory of constrained quantization [15]. This tensor product is defined through the modular theory of operator algebras due to Tomita and Takesaki [21, 11, 7], whose importance in quantum field theory has been particularly empasized by Borchers [3].

The purpose of the present paper is to relate the Connes-Sauvageot tensor product to Morita equivalence for von Neumann algebras. This will be done in a certain categorical setting, which itself was inspired by quantum field theory [14]. Namely, we work in the framework of bicategories $[2,16]$. Introduced by Bénabou in 1967 [2], bicategories form a generalization of monoidal categories (a monoidal category is a bicategory with one onject). Monoidal categories now form a standard tool in the analysis of superselection sectors [8]. Our main result is that von Neumann algebras form a bicategory in which the morphisms are correspondences composed by the above-mentioned tensor product, and that two von Neumann algebras are Morita equivalent iff they are equivalent objects in this bicategory. This insight should have important applications to all areas mentioned.

The notion of Morita equivalence was originally introduced for rings. Two rings $R, S$ are called Morita equivalent when their respective categories of right modules, $\mathfrak{M}_{R}$ and $\mathfrak{M}_{S}$, are equivalent. Rieffel introduced the notion of Morita equivalence for von Neumann algebras [17]. He called two von Neumann algebras $\mathfrak{M}, \mathfrak{N}$ Morita equivalent when their categories of normal unital *-representations, $\operatorname{Rep}(\mathfrak{M})$ and $\operatorname{Rep}(\mathfrak{N})$, are equivalent, where the equivalence is implemented by a normal *-functor. In this paper we will use an equivalent definition: Two von Neumann algebras $\mathfrak{M}, \mathfrak{N}$ are Morita equivalent when a correspondence $\mathfrak{M} \rightarrow \mathcal{H} \leftarrow \mathfrak{N}$ exists for which the representation of $\mathfrak{M}$ on $\mathcal{H}$ is faithful and $\mathfrak{M}^{\prime} \simeq \mathfrak{N}^{o p}$ holds. The goal of this paper is to show a similarity between Morita equivalence for rings and for von Neumann algebras. 
As a tool, we will use bicategories $[2,16]$. Bicategories allow a composition of arrows that is associative only up to a family of isomorphisms. Strictly associative bicategories are 2-categories. For instance, the class of categories as objects, functors as 1-morphisms and natural transformations as 2-morphisms form a 2-category. Another example is the 2-category of topological spaces as objects, continuous maps as 1-morphisms, and homotopy classes of continuous maps as 2-morphisms. Each (relaxed) monoidal category $M$ forms a bicategory, which in general is not a 2-category. This bicategory consists of one object $(M)$; the objects of the category $M$ form 1-morphisms of the bicategory. A composition functor $M \times M \rightarrow M$ that is associative up to isomorphism exists, since $M$ is monoidal. The arrows $M_{1}$ of the category $M$ form the 2-morphisms of the bicategory. The natural isomorphisms that are associated to the monoidal category ascertain that the coherence axioms for a bicategory are satisfied.

Further instructive examples of bicategories are the bicategory [Rings] and the bicategory [W*]. The bicategory [Rings] consists of rings as objects, bimodules as 1-morphisms, and bimodule maps as 2-morphisms. Similarly, but adding the appropriate analytical structure, the bicategory [W*] consists of von Neumann algebras as objects, correspondences as 1-morphisms, and bounded intertwiners as 2-morphisms.

Although from a categorical point of view the fact that Morita equivalence for rings is equivalent to equivalence in the bicategory is straightforward $[2,5,16]$ (and some would say tautological, see c.f. $[22,23])$, the corresponding situation for von Neumann algebras is highly nontrivial for analytical reasons. For example, the fact that the coherence axiom in the definition of a bicategory is satisfied in our case has to be proved with the aid of Tomita-Takesaki modular theory. The same is true for our second main result, which we have already mentioned: two von Neumann algebras are Morita equivalent iff they are equivalent objects in $\left[\mathrm{W}^{*}\right]$.

This article is an abbreviated version of [5]. All routine calculations may be found there, including a simple account of the bicategorical approach to Morita theory for rings.

Section II discusses the case of von Neumann algebras. First, we will prepare for the construction of the bicategory [ $\left.\mathrm{W}^{*}\right]$ of von Neumann algebras. We will use the concept of "Connes fusion" [7, 19] for the composition functor in this bicategory. This terminology is due to Wasserman [24]. Second, we will show that von Neumann algebras indeed form a bicategory. Finally, we will prove that two von Neumann algebras are Morita equivalent iff they are equivalent objects in the bicategory [W*].

\section{THE BICATEGORY OF VON NEUMANN ALGEBRAS}

As a preparation for the main result of this paper, consider the following definition, due to Connes [7].

Definition II.1. Let $\mathfrak{M}, \mathfrak{N}$ be von Neumann algebras, $\mathcal{H}$ a Hilbert space. Suppose $\pi_{l}$ is a normal unital representation of $\mathfrak{M}$ on $\mathcal{H}$ and $\pi_{r}$ is a normal unital representation of $\mathfrak{N}^{\text {op }}$ on $\mathcal{H}$ (or equivalently, an anti-representation of $\mathfrak{N})$ such that the actions of $\pi_{l}(\mathfrak{M})$ and $\pi_{r}(\mathfrak{N})$ commute. The triple $\left[\pi_{l}, \pi_{r}, \mathcal{H}\right]$ is called a correspondence, denoted by $\mathfrak{M} \rightarrow \mathcal{H} \leftarrow \mathfrak{N}$. We write $x \eta$ instead of $\pi_{l}(x) \eta$ and $\eta y$ instead of $\pi_{r}(y) \eta$, for $x \in \mathfrak{M}, \eta \in \mathcal{H}, y \in \mathfrak{N}$.

Viewing a von Neumann algebra as a ring, a correspondence may be seen as a bimodule. Examples of correspondences are not difficult to find. Suppose we have a normal, unital representation of a von Neumann algebra $\mathfrak{M}$ on a Hilbert space $\mathcal{H}$. Then we immediately have a correspondence

$$
\mathfrak{M} \rightarrow \mathcal{H} \leftarrow\left(\mathfrak{M}^{\prime}\right)^{o p}
$$

We will use the following notation. Let $\phi$ be a faithful normal semifinite weight on a von Neumann algebra $\mathfrak{M}$. Let $\mathfrak{M}_{\phi}=\left\{x \in \mathfrak{M} \mid \phi\left(x^{*} x\right)<\infty\right\}$ and let $\mathcal{H}_{\phi}$ be the Hilbert space, formed by completion of $\mathfrak{M}_{\phi}$ in the inner product originating from $\phi$. The canonical inclusion will be denoted by $\Lambda_{\phi}$ : $\mathfrak{M}_{\phi} \rightarrow \mathcal{H}_{\phi}$. The associated modular conjugation and modular operator will be denoted by $J_{\phi}$ and $\Delta_{\phi}$, respectively. The specific choice of $\phi$ merely affects these data, known as a standard form of $\mathfrak{M}$, up to unitary equivalence [10]. 
Definition II.2. An identity correspondence of a von Neumann algebra $\mathfrak{M}$ is given by a Hilbert space $\mathcal{H}_{\phi}$ as above, with left representation $\pi_{l}$ and right representation $\pi_{r}$ defined on the dense subspace $\Lambda_{\phi}\left(\mathfrak{M}_{\phi}\right) \subset \mathcal{H}_{\phi}$ by (II.2) and (II.3).

$$
\begin{aligned}
\pi_{l}: \mathfrak{M} & \longrightarrow \mathcal{B}\left(\mathcal{H}_{\phi}\right) ; \\
\pi_{l}(x) \Lambda_{\phi}(\eta) & =\Lambda_{\phi}(x \eta)
\end{aligned}
$$

and

$$
\begin{aligned}
\pi_{r}: \mathfrak{M} & \longrightarrow \mathcal{B}\left(\mathcal{H}_{\phi}\right) \\
\pi_{r}(x) \Lambda_{\phi}(\eta) & =J_{\phi} \pi_{l}\left(x^{*}\right) J_{\phi} \Lambda_{\phi}(\eta) .
\end{aligned}
$$

We will subsequently omit $\Lambda_{\phi}$ when no confusion arises.

Note that an identity correspondence is a special case of (II.1), since we have $J_{\phi} \mathfrak{M} J_{\phi} \cong \mathfrak{M}^{\prime}$ by Tomita-Takesaki theory. Since the standard form of a von Neumann algebra is unique up to unitary equivalence [10], so is an identity correspondence. Hence we will often write

$$
\mathfrak{M} \rightarrow \mathfrak{L}^{2}(\mathfrak{M}) \leftarrow \mathfrak{M},
$$

for an identity correspondence, suppressing the weight $\phi$. The notation $\mathfrak{L}^{2}(\mathfrak{M})$ is chosen in analogy to measure theory; recall that the latter is considered to be the commutative version of von Neumann algebras. For $\mathfrak{M}=\mathfrak{L}^{\infty}(X, \mu)$ one has $\mathfrak{L}^{2}(\mathfrak{M}) \simeq \mathfrak{L}^{2}(X, \mu)$.

As a preparation for the construction of the bicategory $\left[\mathrm{W}^{*}\right]$, we will now review the concept of Connes fusion, or the relative tensor product, see $[7,6,19]$.

Let $\mathfrak{M} \rightarrow \mathcal{H} \leftarrow \mathfrak{N}$ and $\mathfrak{N} \rightarrow \mathcal{K} \leftarrow \mathfrak{P}$ be two correspondences. Let $\phi$ be an arbitrary faithful normal semifinite weight on $\mathfrak{N}$. To obtain the relative tensor product $\mathfrak{M} \rightarrow \mathcal{H} \otimes_{\mathfrak{N}} \mathcal{K} \leftarrow \mathfrak{P}$, one defines a form $(,)_{0}$ on the algebraic tensor product $D(\mathcal{H}, \phi) \otimes_{\mathbb{C}} \mathcal{K}$, quotients by the null space, and completes. Here $D(\mathcal{H}, \phi)$ is the dense subspace of $\phi$-bounded vectors of $\mathcal{H}$, defined by

$$
D(\mathcal{H}, \phi)=\left\{\eta \in \mathcal{H} \mid \exists c: \forall y \in \mathfrak{N}_{\phi},\left\|\pi_{r}(y) \eta\right\|_{\mathcal{H}} \leq c \phi\left(y^{*} y\right)\right\}
$$

Equivalently, one can define $D(\mathcal{H}, \phi)$ as the set of $\eta \in \mathcal{H}$ for which the operator $R_{\eta}^{\phi}: \mathcal{H}_{\phi} \rightarrow \mathcal{H}$, is bounded, where $R_{\eta}^{\phi}$ is defined on the dense subspace $\mathfrak{N}_{\phi} \subset \mathfrak{N}$ by

$$
R_{\eta}^{\phi}\left(J_{\phi} \Lambda_{\phi}\left(y^{*}\right)\right)=\pi_{r}(y) \eta, \quad \forall y \in \mathfrak{N}_{\phi}
$$

Recall that $\mathcal{H}_{\phi}$ is a standard Hilbert space of $\mathfrak{N}$.

For later use, we will state some properties of $R_{\eta}$. The proof is easy calculation. For all $\eta_{1}, \eta_{2} \in$ $D(\mathcal{H}, \phi), x \in \mathfrak{N}$, we have

$$
\begin{aligned}
& R_{\eta_{1}}^{*} R_{\eta_{2}} \in \mathfrak{N} . \\
& \Delta^{-1 / 2} x^{*} \Delta^{1 / 2} R_{\eta_{1}}^{*} R_{\eta_{2}}=R_{\eta_{1} x}^{*} R_{\eta_{2}} \text { on } \mathfrak{L}^{2}(\mathfrak{N}) \\
& R_{\eta_{1}}^{*} R_{\eta_{2}} \Delta^{1 / 2} x \Delta^{-1 / 2}=R_{\eta_{1}}^{*} R_{\eta_{2} x} \text { on } \mathfrak{L}^{2}(\mathfrak{N}) .
\end{aligned}
$$

Further, we need a subspace $\mathfrak{N}_{0} \subset \mathfrak{N}$ that will be used later (Remark II.4). $\mathfrak{N}_{0}$ is defined as follows. Let $\tau_{t}(x)=\Delta_{\phi}^{i t} x \Delta_{\phi}^{-i t}, x \in \mathfrak{N}$, be the modular automorphism group of $\mathfrak{N}$, let

$$
x_{n}=\sqrt{n / \pi} \int d t e^{-n t^{2}} \tau_{t}(x) \quad n=1,2, \ldots
$$

and finally put

$$
\mathfrak{N}_{0}=\operatorname{Span}\left\{x_{n} \mid x \in \mathfrak{N}_{\phi}, n=1,2, \ldots\right\} .
$$


Lemma II.3. In the above notation, $x_{n} \rightarrow x \sigma$-weakly. Moreover, $\mathfrak{N}_{0}$ is a $\sigma$-dense and norm-dense subspace of $\mathfrak{N}_{\phi}$. Further, for $\xi \in \mathfrak{N}_{0}$,

$$
\Delta^{1 / 2} \xi \Delta^{-1 / 2} \in \mathfrak{N}_{\phi}
$$

Proof. Cf. [5] for details. The proof follows from the analyticity of the elements of $\mathfrak{N}_{0}$, see [4], and positivity and modular invariance of the weight $\phi$. See also [21].

To detail, the sesquilinear form $(,)_{0}$ on $D(\mathcal{H}, \phi) \otimes_{\mathbb{C}} \mathcal{K}$ is defined by

$$
\left(\eta_{1} \otimes \zeta_{1}, \eta_{2} \otimes \zeta_{2}\right)_{0}:=<\zeta_{1}, R_{\eta_{1}}^{*} R_{\eta_{2}} \zeta_{2}>_{\mathcal{K}}
$$

where $<,>_{\mathcal{K}}$ is the inner product on the Hilbert space $\mathcal{K}$. Note that since $R_{\eta_{1}}^{*} R_{\eta_{2}}$ is an element of $\mathfrak{N}$ by (II.4), the second argument of the inner product is indeed an element of $\mathcal{K}$. It is easy to see that $(,)_{0}$ is a pre-inner product. Hence, if we quotient by the null space $\mathcal{N}$ and complete, we obtain a Hilbert space, denoted by $\mathcal{H} \otimes_{\mathfrak{N}} \mathcal{K}$. This Hilbert space $\mathcal{H}{\bigotimes_{\mathfrak{N}}}_{\mathcal{K}}$ is an $\mathfrak{M} \rightarrow \mathcal{H} \otimes_{\mathfrak{N}} \mathcal{K} \leftarrow \mathfrak{P}$ correspondence, so that we may regard the above construction as the fusion of correspondences rather than merely as Hilbert spaces. Namely, we let the representations of $\mathfrak{M}$ on $\mathcal{H}$ and of $\mathfrak{P}$ on $\mathcal{K}$ descend to the quotient. Routine verification will show that the null space is closed under the actions of $\mathfrak{M}$ and $\mathfrak{P}$.

Remark II.4. The relative tensor product $\nabla_{\mathfrak{N}}$ has a property analogous to the 'balancedness' of the tensor product of bimodules over rings, but with a crucial and interesting modification. Balancedness for a ring $R$ and bimodules $M_{R},{ }_{R} N$ means we have $\left(m r \otimes_{R} n\right)=\left(m \otimes_{R} r n\right)$, for $m \in M_{R}, n \in_{R}$ $N, r \in R$. However, for $\eta \in \mathcal{H}, n \in \mathfrak{N}_{0}$, and $\zeta \in \mathcal{K}$ we have

$$
\eta n \otimes_{\mathfrak{N}} \zeta=\eta \otimes_{\mathfrak{N}}\left(\Delta^{1 / 2} n \Delta^{-1 / 2}\right) \zeta
$$

and

$$
\eta \bigotimes_{\mathfrak{N}} n \zeta_{1}=\eta\left(\Delta^{-1 / 2} n \Delta^{1 / 2}\right) \bigotimes_{\mathfrak{N}} \zeta
$$

For let $\eta_{2} \in D(\mathcal{H}, \phi), x \in \mathfrak{N}_{0}, \zeta_{2} \in \mathcal{K} ;$ then

$$
\begin{aligned}
\left(\eta_{1} x \otimes \zeta_{1}, \eta_{2} \otimes \zeta_{2}\right)_{0}= & <\zeta_{1}, R_{\eta_{1} x}^{*} R_{\eta_{2}} \zeta_{2}>_{\mathcal{K}} \\
= & <\zeta_{1},\left(\Delta^{-1 / 2} x^{*} \Delta^{1 / 2}\right) R_{\eta_{1}}^{*} R_{\eta_{2}} \zeta_{2}>_{\mathcal{K}} \\
= & <\left(\Delta^{1 / 2} x \Delta^{-1 / 2}\right) \zeta_{1}, R_{\eta_{1}}^{*} R_{\eta_{2}} \zeta_{2}>_{\mathcal{K}} \\
= & \left(\eta_{1} \otimes\left(\Delta^{1 / 2} x \Delta^{-1 / 2}\right) \zeta_{1}, \eta_{2} \otimes \zeta_{2}\right)_{0}
\end{aligned}
$$

where the second equality holds because of (II.5). This implies that $\left(\eta_{1} x \otimes \zeta_{1}\right)-\left(\eta_{1} \otimes\left(\Delta^{1 / 2} x \Delta^{-1 / 2}\right) \zeta_{1}\right)$ belongs to the null space $\mathcal{N}$. Since $\mathfrak{N}_{0}$ is a dense subspace of $\mathfrak{N}_{\phi}$, (II.7) holds for the completion $\mathcal{H} \otimes_{\mathfrak{N}} \mathcal{K}$. Moreover, for $x \in \mathfrak{N}_{0}$, we have $\Delta^{1 / 2} x \Delta^{-1 / 2} \in \mathfrak{N}$ so that all expressions in (II.9) are defined. The proof of (II.8) follows by a similar argument.

Now, we will show that the collection of von Neumann algebras forms a bicategory. This result was already stated in [14] without proof.

Proposition II.5. For any two von Neumann algebra's $\mathfrak{M}, \mathfrak{N}$, let $(\mathfrak{M}, \mathfrak{N})$ be the category of correspondences as objects, and bounded linear bimodule maps as arrows. Then there exists a bicategory $\left[\mathrm{W}^{*}\right]$ with von Neumann algebras as objects and correspondences as 1-morphisms. The composition functor $(\mathfrak{M}, \mathfrak{N}) \times(\mathfrak{N}, \mathfrak{P}) \rightarrow(\mathfrak{M}, \mathfrak{P})$ is given by the relative tensor product $\bigotimes_{\mathfrak{N}}$, and the unit arrow in $(\mathfrak{M}, \mathfrak{M})$ is given by $I_{\mathfrak{M}}=\mathfrak{M} \rightarrow \mathfrak{L}^{2}(\mathfrak{M}) \leftarrow \mathfrak{M}$. 
Proof. Unlike the case of rings, a nontrivial issue arises in the verification, related to Remark II.4. We will only stress the points at which the verification is not straightforward. Note that the unit arrow $I_{\mathfrak{M}}$ is unique up to unitary equivalence, as was mentioned after Definition II.2. The natural isomorphism to obtain associativity is defined element wise and hence the existence of associativity coherence is routine calculation. The difficulty lies in the construction of the left and right identities and identity coherence.

For each pair of von Neumann algebras $(\mathfrak{M}, \mathfrak{N})$, the so-called left-identity $L_{(\mathfrak{M}, \mathfrak{N})}$ is a natural isomorphism between the functors $\mathfrak{L}^{2}(\mathfrak{M}) \nabla_{\mathfrak{M}}(-)$ and the identity functor $\operatorname{Id}_{(\mathfrak{M}, \mathfrak{N})}$ from $(\mathfrak{M}, \mathfrak{N})$ to $(\mathfrak{M}, \mathfrak{N})$. More concretely, recall that $\Lambda_{\phi}: \mathfrak{M}_{\phi} \rightarrow \mathfrak{L}^{2}(\mathfrak{M})$ denotes the inclusion map. It satisfies

$$
\begin{aligned}
x \Lambda_{\phi}(y) & =\Lambda_{\phi}(x y) \\
\Delta_{\phi}^{1 / 2} \Lambda_{\phi}(y) & =\Lambda_{\phi}\left(\Delta_{\phi}^{1 / 2} y \Delta_{\phi}^{-1 / 2}\right),
\end{aligned}
$$

for $x \in \mathfrak{M}, y \in \mathfrak{M}_{0}$. See [11].

Let $x \in \mathfrak{M}_{0}, \zeta \in \mathcal{K} \in(\mathfrak{M}, \mathfrak{N})$. Then we define $L_{(\mathfrak{M}, \mathfrak{N})}$ on the dense subspace $\mathfrak{M}_{0} \bigotimes_{\mathfrak{M}} \mathcal{K}$ by

$$
L_{(\mathfrak{M}, \mathfrak{N})}: \Lambda_{\phi}(x) \bigotimes_{\mathfrak{M}} \zeta \mapsto x \zeta .
$$

We will show that the map (II.11) is continuous, so that we may extend it to $\mathfrak{L}^{2}(\mathfrak{M}) \bigotimes_{\mathfrak{M}} \mathcal{K}$. Consider

$$
\left\|\Lambda_{\phi}(x) \bigotimes_{\mathfrak{M}} \zeta\right\|^{2}=<\zeta, R_{\Lambda_{\phi}(x)}^{*} R_{\Lambda_{\phi}(x)} \zeta>_{\mathcal{K}} .
$$

Observe that in this case,by definition of the right representation of $\mathfrak{M}$ on $\mathfrak{L}^{2}(\mathfrak{M})$, the operator $R_{\Lambda_{\phi}(x)}: \mathfrak{L}^{2}(\mathfrak{M}) \rightarrow \mathfrak{L}^{2}(\mathfrak{M})$ is given by

$$
R_{\Lambda_{\phi}(x)} J_{\phi} \Lambda_{\phi}\left(y^{*}\right)=\pi_{r}(y) \Lambda_{\phi}(x)=J_{\phi} y^{*} J_{\phi} \Lambda_{\phi}(x),
$$

on the dense subspace $\mathfrak{M}_{0}$.

The following claims lead to continuity of the map (II.11). The proofs are easy calculations.

- For $x, z \in \mathfrak{M}_{0}$, we have $R_{\Lambda_{\phi}(x)} \Lambda_{\phi}(z)=\Lambda_{\phi}(x z)$.

- For $x \in \mathfrak{M}_{0}$, the operator $R_{\Lambda_{\phi}(x)}^{*} R_{\Lambda_{\phi}(x)}$ equals $\Lambda_{\phi}\left(x^{*} x\right)$ as operators on $\mathfrak{L}^{2}(\mathfrak{M})$.

Combining the statements above we obtain

$$
\left\|\Lambda_{\phi}(x) \bigotimes_{\mathfrak{M}} \zeta\right\|^{2}=<\zeta, R_{\Lambda_{\phi}(x)}^{*} R_{\Lambda_{\phi}(x)} \zeta>_{\mathcal{K}}=\|x \zeta\|_{\mathcal{K}}^{2}
$$

Hence $\Lambda_{\phi}(x) \bigotimes_{\mathfrak{M}} \zeta \mapsto x \zeta$ is a continuous map. It remains to show that $L_{(\mathfrak{M}, \mathfrak{N})}$ is unitary and hence a natural isomorphism. We know that it is isometric by (II.12). Hence it is sufficient to show that the image of $\mathfrak{M}_{0} \otimes \mathcal{K}$ is dense in $\mathcal{K}$. Generally, $\mathbb{1} \in \mathfrak{M}$ is not an element of $\mathfrak{M}_{\phi}$. But, since $\mathfrak{M}_{0}$ is norm-dense in $\mathfrak{M}$, we may find a net $\mathcal{E}_{\alpha}$ in $\mathfrak{M}_{0}$, converging in norm to $\mathbb{1}$. Let $\zeta \in \mathcal{K}$. Then

$$
\left\|\mathcal{E}_{\alpha} \zeta-\mathbb{1} \zeta\right\|_{\mathcal{K}} \leq\left\|\mathcal{E}_{\alpha}-\mathbb{1}\right\|_{\mathfrak{M}} \cdot\|\zeta\|_{\mathcal{K}} \longrightarrow 0
$$

where we used the fact that a representation is norm decreasing. Hence, $\mathfrak{M}_{0} \mathcal{K}$ is dense in $\mathcal{K}$.

In contrast to the case of rings, the right identity $R_{(\mathfrak{M}, \mathfrak{N})}$ is defined differently from the left identity. The right identity should be a natural isomorphism between the functor $(-) \bigotimes_{\mathfrak{N}} \mathfrak{L}^{2}(\mathfrak{N})$ and the identity $I d_{(\mathfrak{M}, \mathfrak{N})}$. Let $\mathcal{H} \in(\mathfrak{M}, \mathfrak{N}), \eta \in D(\mathcal{H}, \phi), y \in \mathfrak{N}_{0}$. Then we define $R_{(\mathfrak{M}, \mathfrak{N})}$ on the dense subspace $D(\mathcal{H}, \phi) \otimes_{\mathfrak{N}} \mathfrak{N}_{0}$ by

$$
R_{(\mathfrak{M}, \mathfrak{N})} \quad: \quad \eta \bigotimes_{\mathfrak{N}} \Lambda_{\phi}(y) \quad \mapsto \quad \eta\left(\Delta_{\phi}^{-1 / 2} y \Delta_{\phi}^{1 / 2}\right)
$$


Note that $\Delta_{\phi}^{-1 / 2} y \Delta_{\phi}^{1 / 2} \in \mathfrak{N}$, for $y \in \mathfrak{N}_{0}$, so that $\eta\left(\Delta_{\phi}^{-1 / 2} y \Delta_{\phi}^{1 / 2}\right)$ is defined by definition of the right representation of $\mathfrak{N}$ on $\mathcal{H}$. We will show next that the map (II.13) is continuous, so that we may extend it to $\mathcal{H} \otimes_{\mathfrak{N}} \mathfrak{L}^{2}(\mathfrak{N})$. Consider

$$
\left\|\eta \bigotimes_{\mathfrak{N}} \Lambda_{\phi}(y)\right\|^{2}=\left(\Lambda_{\phi}(y), R_{\eta}^{*} R_{\eta} \Lambda_{\phi}(y)\right)_{\mathfrak{L}^{2}(\mathfrak{N})} .
$$

In this case, the operator $R_{\eta}: \mathfrak{L}^{2}(\mathfrak{N}) \rightarrow \mathcal{H}$ is defined by

$$
R_{\eta} J_{\phi} \Lambda_{\phi}\left(y^{*}\right)=\pi_{r}(y) \eta=\eta y,
$$

on the dense subspace $\mathfrak{N}_{0}$.

We make use of the following fact, proven by Connes ( $[6$, Lemma 4]). For $\zeta \in D(\mathcal{H}, \phi)$, we have

$$
\phi\left(R_{\zeta}^{*} R_{\zeta}\right)=\|\zeta\|_{\mathcal{H}}^{2} .
$$

Hence, using (II.5) and (II.6), we obtain

$$
\begin{aligned}
\left\|\eta \otimes_{\mathfrak{N}} \Lambda_{\phi}(y)\right\|^{2} & =\left(\Lambda_{\phi}(y), R_{\eta}^{*} R_{\eta} \Lambda_{\phi}(y)\right)_{\mathfrak{L}^{2}(\mathfrak{N})} \\
& =\phi\left(y^{*} R_{\eta}^{*} R_{\eta} y\right) \\
& \left.=\phi\left(R_{\eta\left(\Delta_{\phi}^{*}\right.}^{*} y \Delta_{\phi}^{1 / 2}\right) R_{\eta\left(\Delta_{\phi}^{-1 / 2} y \Delta_{\phi}^{1 / 2}\right)}\right) \\
& =\left\|\eta\left(\Delta_{\phi}^{-1 / 2} y \Delta_{\phi}^{1 / 2}\right)\right\|_{\mathcal{H}}^{2} .
\end{aligned}
$$

Hence $\eta \nabla_{\mathfrak{N}} \Lambda_{\phi}(y) \mapsto \eta\left(\Delta_{\phi}^{1 / 2} y \Delta_{\phi}^{-1 / 2}\right)$ is a continuous map. It is left to show that $R_{(\mathfrak{M}, \mathfrak{N})}$ is unitary and hence a natural isomorphism. As above, (II.14) shows that it is isometric, hence it is sufficient to show that the image of $D(\mathcal{H}, \phi) \otimes \mathfrak{N}_{0}$ is dense in $\mathcal{H}$. As before, we have a net $\mathcal{E}_{\alpha}$ in $\mathfrak{N}_{0}$ converging to 1 . Consider the net $\Delta_{\phi}^{1 / 2} \mathcal{E}_{\alpha} \Delta_{\phi}^{-1 / 2}$. This net is contained in $\mathfrak{N}_{\phi}$, as follows from Lemma II.3. By the inclusion $\mathfrak{N}_{0} \subset \mathfrak{N}_{\phi} \subset \mathfrak{L}^{2}(\mathfrak{N})$ and the continuity just proven, we have

$$
\Delta_{\phi}^{1 / 2} \mathcal{E}_{\alpha} \Delta_{\phi}^{-1 / 2} \otimes_{\mathfrak{N}} \eta \longmapsto \eta \mathcal{E}_{\alpha},
$$

for $\eta \in D(\mathcal{H}, \phi)$. The right hand side converges to $\eta$ in norm. The observation that $D(\mathcal{H}, \phi) \subset \mathcal{H}$ is a dense subspace finishes the proof.

Finally, we need to prove identity coherence. Let $\mathfrak{M}, \mathfrak{N}, \mathfrak{P}$ be von Neumann algebras and $\mathfrak{M} \mathcal{H}_{\mathfrak{N}}$ and ${ }_{\mathfrak{N}} \mathcal{K}_{\mathfrak{P}}$ associated correspondences. It will be shown that the following diagram commutes:

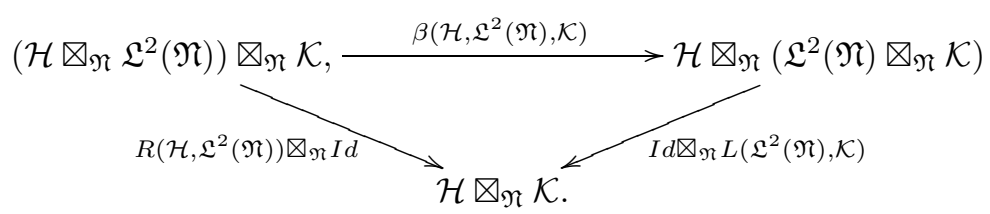

Since the upper arrow $\beta\left(\mathcal{H}, \mathfrak{L}^{2}(\mathfrak{N}), \mathcal{K}\right)$ is the associativity isomorphism, identity coherence boils down to

$$
\left(\eta\left(\Delta_{\phi}^{-1 / 2} n \Delta_{\phi}^{1 / 2}\right) \bigotimes_{\mathfrak{N}} \zeta\right) \cong\left(\eta \otimes_{\mathfrak{N}} n \zeta\right),
$$

for $\eta \in \mathcal{H}, n \in \mathfrak{L}^{2}(\mathfrak{N}), \zeta \in \mathcal{K}$. This follows immediately from Remark II.4.

Note that the bicategory of von Neumann algebras, constructed as above, depends on the choice of a faithful normal semifinite weight for each von Neumann algebra, because the definition of the composition functor (i.e. the relative tensor product) depends on this choice. However, different weights lead to unitarily equivalent relative tensor products [19]. Hence the bicategory depends on the chosen weights only up to isomorphism. 


\section{MORITA THEORY FOR VON NEUMANN ALGEBRAS}

The notion of Morita equivalence for von Neumann algebras was originally introduced by Rieffel. Two von Neumann algebras $\mathfrak{M}, \mathfrak{N}$ are said to be Morita equivalent if their representation categories $\operatorname{Rep}(\mathfrak{M})$ and $\operatorname{Rep}(\mathfrak{N})$ are equivalent, where the equivalence is implemented by a normal *-functor. Here $\operatorname{Rep}(\mathfrak{M})$ denotes the category of normal unital *-representations on Hilbert spaces as objects, and bounded linear intertwiners as arrows. For our purposes, we will use the following (equivalent) definition.

Definition III.1. Two von Neumann algebras $\mathfrak{M}, \mathfrak{N}$ are called Morita equivalent, if a correspondence $\mathfrak{M} \rightarrow \mathcal{H} \leftarrow \mathfrak{N}$ exists, where the representation of $\mathfrak{M}$ on $\mathcal{H}$ is faithful and for which

$$
\mathfrak{M}^{\prime} \simeq \mathfrak{N}^{o p}
$$

Note that faithfulness of $\mathfrak{M}$ directly implies faithfulness for $\mathfrak{N}$. Using somewhat different notation, Rieffel, [17, Thm 8.5] proves equivalence between the definition above and his original definition. In this paper, we will not follow Rieffel's approach, which involves so-called normal $\mathfrak{N}$-rigged $\mathfrak{M}$-modules, and interior tensor products of these modules. Rieffel proves an Eilenberg-Watts like theorem ( [17, Thm. 5.5]), which states that all functors of $\operatorname{Rep}(\mathfrak{N})$ to $\operatorname{Rep}(\mathfrak{M})$ are equivalent to taking the tensor product with such a normal $\mathfrak{N}$-rigged $\mathfrak{M}$-module. However, these modules are not Hilbert spaces, which is what we would like considering our definition of the tensor product and the bicategory of von Neumann algebras.

Now we are ready for the main theorem.

Theorem III.2. Two von Neumann algebras are equivalent objects in the bicategory [W*] iff they are Morita equivalent.

Proof. First, let us reformulate the first property in the theorem. The property that two von Neumann algebras $\mathfrak{M}, \mathfrak{N}$ are equivalent objects in the bicategory means that there must be an arrow in $(\mathfrak{M}, \mathfrak{N})$, i.e., a correspondence $\mathfrak{M} \rightarrow \mathcal{H} \leftarrow \mathfrak{N}$ that is invertible up to isomorphism. In other words, there exists an arrow $\mathfrak{N} \rightarrow \mathcal{H}^{-1} \leftarrow \mathfrak{M}$ in $(\mathfrak{N}, \mathfrak{M})$, such that

$$
\begin{aligned}
\mathfrak{M} & \rightarrow \mathcal{H} \otimes_{\mathfrak{N}} \mathcal{H}^{-1} \leftarrow \mathfrak{M} \cong \mathfrak{M} \rightarrow \mathfrak{L}^{2}(\mathfrak{M}) \leftarrow \mathfrak{M} \text { in }(\mathfrak{M}, \mathfrak{M}), \\
\mathfrak{N} & \rightarrow \mathcal{H}^{-1} \bigotimes_{\mathfrak{M}} \mathcal{H} \leftarrow \mathfrak{N} \cong \mathfrak{N} \rightarrow \mathfrak{L}^{2}(\mathfrak{N}) \leftarrow \mathfrak{N} \text { in }(\mathfrak{N}, \mathfrak{N}) .
\end{aligned}
$$

"६:" Let $\mathfrak{M}, \mathfrak{N}$ be Morita equivalent. Then we have a correspondence

$\mathfrak{M} \rightarrow \mathcal{H} \leftarrow \mathfrak{N}$, where the representation of $\mathfrak{M}$ on $\mathcal{H}$ is faithful, and $\mathfrak{M}^{\prime} \simeq \mathfrak{N}^{o p}$. From $\mathfrak{M} \rightarrow \mathcal{H} \leftarrow \mathfrak{N}$, we can define a correspondence $\mathfrak{N} \rightarrow \overline{\mathcal{H}} \leftarrow \mathfrak{M}$ by

$$
n \bar{\eta} m:=m^{*} \eta n^{*}, \quad \text { for } n \in \mathfrak{N}, m \in \mathfrak{M}, \eta \in \mathcal{H},
$$

where $\overline{\mathcal{H}}$ is $\mathcal{H}$ as a set, with the addition operator of $\mathcal{H}$ and conjugate scalar multiplication and inner product. Sauvageot [19, Prop. 3.1] proves that the induced representation of $\mathfrak{M}$ on the relative tensor product $\mathcal{H} \otimes_{\mathfrak{N}} \overline{\mathcal{H}}$ is in standard form, hence

$$
\mathfrak{M} \rightarrow \mathcal{H} \otimes_{\mathfrak{N}} \overline{\mathcal{H}} \leftarrow \mathfrak{M} \cong \mathfrak{M} \rightarrow \mathfrak{L}^{2}(\mathfrak{M}) \leftarrow \mathfrak{M}
$$

Applying the same reasoning to $\mathfrak{N} \rightarrow \overline{\mathcal{H}} \leftarrow \mathfrak{M}$, we obtain $\mathfrak{M} \rightarrow \overline{\overline{\mathcal{H}}} \leftarrow \mathfrak{N}$ and clearly, we have $\overline{\overline{\mathcal{H}}}=\mathcal{H}$. Then

$$
\begin{aligned}
\mathfrak{N} \rightarrow \overline{\mathcal{H}} \otimes_{\mathfrak{M}} \overline{\overline{\mathcal{H}}} \leftarrow \mathfrak{N} & =\mathfrak{N} \rightarrow \overline{\mathcal{H}} \otimes_{\mathfrak{M}} \mathcal{H} \leftarrow \mathfrak{N} \\
& \cong \mathfrak{N} \rightarrow \mathfrak{L}^{2}(\mathfrak{N}) \leftarrow \mathfrak{N} .
\end{aligned}
$$

Together, (III.3) and (III.4) prove that ${ }_{\mathfrak{M}} \mathcal{H}_{\mathfrak{N}}$ is invertible, its inverse being $\mathfrak{\mathfrak { N }} \overline{\mathcal{H}}_{\mathfrak{M}}$. 
" $\Rightarrow$ :" Suppose we have an invertible correspondence $\mathfrak{M} \rightarrow \mathcal{H} \leftarrow \mathfrak{N}$. We need to show that $\mathfrak{M} \simeq$ $\left(\mathfrak{N}^{o p}\right)^{\prime}$, and that the representation of $\mathfrak{M}$ is faithful. By definition of a correspondence, we have

$$
\mathfrak{M} \subseteq\left(\mathfrak{N}^{o p}\right)^{\prime}
$$

so, considering the representation of $\mathfrak{M}$ on $\mathcal{H} \otimes_{\mathfrak{N}} \mathcal{H}^{-1}$, one has

$$
\mathfrak{M} \otimes i d_{\mathcal{H}^{-1}} \subseteq\left(\mathfrak{N}^{o p}\right)^{\prime} \otimes i d_{\mathcal{H}^{-1}}
$$

Now we will use a result from Sauvageot [19, Prop. 3.3], who shows that for a von Neumann algebra $\mathfrak{P}$ and representations $\mathcal{K}_{1} \leftarrow \mathfrak{P}$ and $\mathfrak{P} \rightarrow \mathcal{K}_{2}$, one has

$$
\left(\mathfrak{P}^{o p}\right)^{\prime} \otimes_{\mathfrak{P}} i d_{\mathcal{K}_{2}}=\left[i d_{\mathcal{K}_{1}} \otimes_{\mathfrak{P}} \mathfrak{P}^{\prime}\right]^{\prime}
$$

in $\mathcal{K}_{1} \otimes_{\mathfrak{P}} \mathcal{K}_{2}$. Applying (III.7) to (III.6) we obtain

$$
\mathfrak{M} \otimes_{\mathfrak{N}} i d_{\mathcal{H}^{-1}} \subseteq\left(\mathfrak{N}^{o p}\right)^{\prime} \otimes_{\mathfrak{N}} i d_{\mathcal{H}^{-1}}=\left[i d_{\mathcal{H}} \otimes_{\mathfrak{N}} \mathfrak{N}^{\prime}\right]^{\prime}
$$

hence, using the commutant of (III.7),

$$
i d_{\mathcal{H}} \otimes_{\mathfrak{N}} \mathfrak{N}^{\prime} \subseteq\left[\mathfrak{M} \otimes_{\mathfrak{N}} i d_{\mathcal{H}^{-1}}\right]^{\prime}=i d_{\mathcal{H}} \otimes_{\mathfrak{N}} \mathfrak{M}^{o p}
$$

Now (III.8) implies that $\mathfrak{N}^{\prime} \subseteq \mathfrak{M}^{o p}$ by the definition of the representation of $\mathfrak{N}^{\prime}$ on $\mathcal{H}_{\mathfrak{N}} \mathcal{H}^{-1}$. This, together with (III.5), proves $\mathfrak{M} \simeq\left(\mathfrak{N}^{o p}\right)^{\prime}$. It remains to be shown that the representation of $\mathfrak{M}$ on $\mathcal{H}$ is faithful. However, this follows immediately from (III.1) and the fact that the standard representation of $\mathfrak{M}$ on $\mathfrak{L}^{2}(\mathfrak{M})$ is faithful.

It is possible to restate Theorem III.2 in terms of representation categories. In the light of the remarks after Definition III.1, the proof follows from Rieffel [17], but we prefer to prove the corollary directly.

Corollary III.3. Two von Neumann algebras are equivalent objects in the bicategory [W*] iff their representation categories are equivalent, where the equivalence is implemented by a normal ${ }^{*}$-functor.

Proof. " $\Rightarrow: "$ The construction of the equivalence functor, given the invertible correspondence, is completely analogous to the case of rings, c.f. $[12,14]$. The fact that this construction yields a ${ }^{*}$-functor follows from a trivial computation. An application of [17, Prop. 7.3] shows that this functor is normal.

"६:" Suppose $F: \operatorname{Rep}(\mathfrak{N}) \rightarrow \operatorname{Rep}(\mathfrak{M})$ is a normal *-functor, implementing the categorical equivalence. Consider $F\left(\mathfrak{L}^{2}(\mathfrak{M})\right.$ ), which has a left $\mathfrak{N}$-action by definition, and a right $\mathfrak{M}$-action through $F$. Applying the Eilenberg-Watts like theorem stated by Sauvageot [19, Prop. 5.3], shows that $F\left(\mathfrak{L}^{2}(\mathfrak{M})\right)$ is invertible. C.f. also Rieffel [17, Prop. 5.4, Thm 5.5].

\section{ACKNOWLEDGMENTS}

This article is based on a conjecture by N.P.Landsman. The author thanks him for his advice and helpful suggestions. 


\section{References}

1. H. Bass, Algebraic K-theory, Benjamin, New York, 1968.

2. J. Bénabou, "Introduction to bicategories," Lecture Notes in Mathematics 47 (1967) 1-77.

3. H. J. Borchers, "On revolutionizing quantum field theory with Tomita's modular theory," J. Math. Phys. 41 (2000) 3604-3673.

4. O. Bratteli, D.W. Robinson, Operator Algebras and Quantum Statistical Mechanics I, SpringerVerlag, New York, 1979.

5. R.M. Brouwer, A Bicategorical Approach to Morita Equivalence for Rings and von Neumann Algebras, http://front.math.ucdavis.edu/math.OA/0301353 .

6. A. Connes, "On the spatial theory of von Neumann algebras," J. of Functional Analysis 35 (1980), 153-164.

7. A. Connes, Noncommutative Geometry, Academic Press, San Diego, 1994.

8. S. Doplicher, "Abstract compact group duals, operator algebras and quantum field theory," Proc. Int. Congress of Mathematicians, Kyoto 1990, Springer-Verlag, Tokyo, 1991, 1319-1333.

9. C. Faith, Algebra: Rings, Modules and Categories I, Springer-Verlag, Berlin, 1973.

10. U. Haagerup, "The standard form of von Neumann algebras, " Math. Scand. 37 (1975) 271-283.

11. R.V. Kadison, J.R. Ringrose, Fundamentals of the Theory of Operator Algebras II, Advanced Theory, Academic Press, New York, 1986.

12. T.Y. Lam, Lectures on Modules and Rings, Springer-Verlag, New York, 1999.

13. N.P. Landsman, Mathematical Topics Between Classical and Quantum Mechanics, SpringerVerlag, New York, 1998.

14. N.P. Landsman, "Bicategories of operator algebras and Poisson manifolds," Mathematical Physics in Mathematics and Physics: Quantum and Operator Algebraic Aspects, ed. R. Longo, Fields Inst. Comm 30 (2001) 271-286.

15. N.P. Landsman, "Quantized reduction as a tensor product," Quantization of Singular Symplectic Quotients, eds. N.P. Landsman, M. Pflaum, M. Schlichenmaier, Birkhäuser, Basel, 2001, 137-180.

16. S. MacLane, Categories for the Working Mathematician, 2nd ed. Springer-Verlag, New York, 1998.

17. M.A. Rieffel, "Morita equivalence for $C^{*}$-algebras and $W^{*}$-algebras," J. of Pure and Applied 
Algebra 5 (1974), 51-96.

18. M.A. Rieffel, "Noncommutative tori-a case study of noncommutative differentiable manifolds," Contemp. Math. 105 (1990) 191-211.

19. J.L. Sauvageot, "Sur le produit tensoriel relatif d' espaces de Hilbert, " J. of Operator Theory 9 (1983), 237-252.

20. A. Schwarz, "Morita equivalence and duality," Nucl. Phys. B534 (1998) 720-738.

21. Ş. Strătilă, L. Zsidó, Lectures on von Neumann Algebras, Abacus Press, Tunbridge Wells, 1979.

22. R. Street, "Enriched categories and cohomology," Quaestiones Math.6 (1983), 265-283.

23. R. Street, "Ideals,radicals, and structure of additive categories," Applied Categorical Structures 3 (1995), 139-149.

24. A. Wassermann, "Operator algebras and conformal field theory III. Fusion of positive energy representations of $\operatorname{LSU}(N)$ using bounded operators," Invent. Math. 133 (1998) 467-538. 